\title{
Study of planar ice crystal orientations in ice clouds from scanning polarization lidar observations
}

\author{
Vincent Noel \\ Analytical Services and Materials, Hampton, VA USA \\ Kenneth Sassen \\ Geophysical Institute, Univ. of Alaska, Fairbanks, AK USA
}

Submitted to the Journal of Applied Meteorology on April 5 $5^{\text {th }}, 2004$

corresponding author :

Vincent Noel

Mail Stop 435

NASA Langley Research Center

Hampton, VA 23681-2199, USA

v.r.noel@larc.nasa.gov 


\begin{abstract}
This paper presents a study of the orientation of ice crystals in cirrus and midlevel clouds, based on the analysis of several cases of scanning polarization lidar observations. The maximum angle that crystals deviate from the horizontal plane is inferred at consecutive altitude levels, by fitting angle-dependent measurements of linear depolarization ratio and backscattered intensities to a theoretical model with a Gaussian distribution of tilt angles. The average deviation angle is linked to the angular variation of backscatter. A rare observation of so-called Parry-oriented columns is also given to highlight the different backscattering behavior with lidar angle. For planar crystals, two orientation modes are found that depend on cloud temperature. High level cold $\left(<\sim-30^{\circ} \mathrm{C}\right)$ clouds show a maximum deviation angle of $\sim 1.0^{\circ}$, while for warmer $\left(>\sim-20^{\circ} \mathrm{C}\right) \mathrm{mi}-$ dlevel clouds this angle averages $\sim 2.0^{\circ}$. This difference is caused by variations in particle shape and fall attitude that depend on temperature, likely involving a transition from simple plates to more widely fluttering dendrites at the warmer temperatures. Polarization lidar scans are clearly uniquely suited for the study of ice crystal orientations in clouds.
\end{abstract}




\section{Introduction}

Cirrus clouds permanently cover as much as $40 \%$ of the Earth surface (Liou 1986), and as such represent an important part of the global radiative budget (Stephens et al. 1990). Unfortunately, their study is generally difficult due to their high altitude and semi-transparency, and requires development of analysis techniques based on remote sensing. Their unique composition of ice crystals, which can adopt an infinite variety of shapes and sizes, grants them with unusual microphysical and radiative properties. Properties of these crystals are still poorly known, and this leads to inconsistencies in their parametrization in Global Circulation Models. Due to these uncertainties, cirrus clouds remain an important source of errors in climate prediction.

Traditionally, most radiative models of cirrus suppose a random orientation of crystals (e.g. Takano et al. 1992), an assumption shared by most algorithms and analysis techniques applied to ice clouds. However, experimental studies of ice crystal riming properties and some unusual optical displays, such as arcs and sun pillars, has led to the discovery that ice crystals tend to orient themselves horizontally (Ono 1969; Sassen 1980). This phenomenon is difficult to study with current in situ devices, while the platform itself can disturb the particles spatial ordering. Takano and Liou (1989) showed that the presence of oriented ice crystals in a cirrus cloud could increase its hemispherical albedo by as much as $30 \%$. Moreover, particle orientation can lead to high uncertainties in the retrieval of cirrus properties from satellite observations, leading in certain cases to optical depths overestimated by $100 \%$ (Masuda and Ishimoto 2004). As a recent study of polarized radiances from the POLDER satellite radiometer has found evidence for 
oriented crystals in $40 \%$ of observed high clouds, this phenomenon could therefore have a strong influence on the global radiative impact of cirrus clouds (Chepfer et al 1999).

In the present study, observations of horizontally oriented ice crystals using a scanning lidar are analyzed. The deviation of the crystals from the horizontal plane is retrieved as a function of cloud altitude, by fitting the lidar angle-dependent observations with a Gaussian model of crystal tilt angles. Different fall attitude modes are explained by considering the off-zenith angle linear depolarization ratios. In Section 2, the phenomenon of crystal orientation in cirrus is placed in its context and possible observation techniques are discussed. Section 3 briefly describes the lidar dataset. A first case study showing oriented crystals is presented in Section 4 and used to illustrate the analysis process. A singular case of oriented columns is presented in Section 5 to highlight the differences with oriented plates and ensure the two shapes can be identified with certainty. Multiple cases of oriented plates are then studied in Section 6, and results discussed in Section 7.

\section{Oriented crystals in ice clouds}

\section{a Crystal orientation behavior}

The first study of crystal orientation in ice clouds was conducted by Magono (1953), who used stroboscopic observations of particles to show that, under some hydrodynamic conditions, platetype snow crystals fall with their major axis horizontal. Later, Ono (1969) generalized this statement by studying ice crystal riming properties, to conclude that free-falling ice crystals (including plates, columns, and dendrites) in a calm atmosphere orient themselves to present the maximum resistance to the air, with a possible slight oscillation about the equilibrium position. 
Particle size was also shown to have an impact on particle orientation : depending on their Reynolds number, thick plates would adopt a orientation close to horizontal for diameters above $100 \mu \mathrm{m}$, when thin plates would require a diameter above $150 \mu \mathrm{m}$ (Sassen 1980). Since then, it has been shown that turbulence effects could make particles wobble around the horizontal plane (Cho et al. 1981), and theoretical models suggest that smaller particles (diameter of $\sim 30 \mu \mathrm{m}$ ) could also adopt a preferential orientation, but with a wider tilt angle $\left(10-20^{\circ}\right)$ from the horizontal plane (Klett 1995). Early densitometer scans of photographic observations of light pillars in ice clouds (Sassen 1980) showed a Gaussian evolution of light intensities in horizontal cross-sections, leading to the conclusion that crystal tilt angles follow a Gaussian distribution. This hypothesis was later confirmed by further observations compared to numerical simulations (Sassen 1987) and, more recently, by a heuristic model of particle dynamics in turbulence based on their shapes (Klett 1995).

\section{b. Studies of crystals orientation by lidar}

While the first studies of ice crystal orienations in the atmosphere used direct photography (e.g., Sassen, 1980), the lidar polarization technique was soon found to provide quite useful information. Owing to its high sensitivity to optically thin cloud layers, this instrument has been extensively used to study cirrus clouds. Lidars with polarization capabilities emit (usually vertically) a light beam linearly polarized in a given plane. The linear depolarization ratio $\delta$ is

then defined as $\delta=\frac{P_{\perp}}{P_{\|}}$, with $P_{\|}$and $P_{\perp}$ powers returned to the lidar receiver, which are, respectively, parallel and orthogonal to the plane of the power transmitted (Schotland et al. 
1971). This quantity, which is sensitive to the shape and orientation of the particles probed by the lidar, was shown at first to provide unambiguous cloud phase discrimination (Pal and Carswell 1973; Liou and Lahore 1974), and later distinguish between crystal shapes (Sassen 1977).

In the presence of oriented crystals in clouds, the lidar returns display very low values of $\delta$ and high backscattered intensities (Platt 1978). This specific signature can be attributed either to planar crystals with their main faces aligned horizontally, or more rarely to columns with their main axis aligned horizontally and a pair of prism faces parallel to the ground. This phenomenon is distinguished from water cloud backscattering, which also lead to low values of $\delta$, by tilting the lidar pointing direction, or lidar angle. When oriented crystals are present, $\delta$ increases rapidly as the lidar beam moves away from the zenith direction (Platt et al. 1978), a behavior reproduced by simulations (Mishchenko et al. 1997). This has led to several lidar-based studies of crystal orientation, showing for example that horizontally-oriented crystals are present in any temperature range (Thomas et al. 1990). Scanning lidars are the logical progression of this technique, and lead to graphic observations of oriented crystals and particles in clouds (Roy and Bissonnette 1999; Sassen and Takono 2000). With such instruments, the change in $\delta$ can be closely monitored while the lidar incidence angle on the cloud changes continuously. Recently, the angular evolution of the lidar depolarization ratio was shown to contain significant information on the orientation behavior of plate-like crystals (Noel et al. 2002): when plotting $\delta$ as a function of the incidence angle, the inflexion point where the change is fastest (i.e., the derivative reaches a maximum) gives an approximation of the maximum deviation angle of the ice plates. Although it is principally planar ice crystals that produce this angular dependence with lidar, the observations from Sassen and Takano (2000) have shown that rare occurrence of 
horizontally-oriented columns crystals falling with a pair of prism faces parallel to the ground can also generate a backscattering maximum in the zenith direction. However, this condition, which produces the rare Parry arc from refracted sunlight, leads to unique strong lidar depolarization as the lidar is scanned a few degrees off the zenith, and so has a very different signature than horizontally oriented plates crystals.

\section{The FARS Scanning Lidar Dataset}

The present paper uses observations of cirrus and midlevel clouds from the scanning Polarization Diversity Lidar or PDL (Sassen 1994). This dual-wavelength $(0.532$ and $1.06 \mu \mathrm{m})$ lidar records atmospheric profiles of backscattered intensity and depolarization ratio with a maximum resolution of $1.5 \mathrm{~m}$ and a maximum scanning of $5^{\circ} \mathrm{s}^{-1}$. Depolarization ratio is calibrated by taking into account the difference between receiver channel gains, and alignment variations by fixing the depolarization ratio at $\delta \sim 0.02$ in mid- to upper-tropospheric signals believe to contain to significant aerosols or cloud backscattering (Young 1980).

The dataset analyzed here was collected at the Facility for Atmospheric Remote Sensing (FARS, see Sassen and Benson 2001) in Salt Lake City, Utah, between 1994 and 2000. Note that although efforts were made to orient the laser table to the zenith direction for these scans using an electronic level, positioning errors of $0.5-1.0^{\circ}$ can occur due to slight movements of the truck containing the PDL. Of the 37 distinct periods of consecutive $\pm 10^{\circ}$ scans around the zenith direction (at a $1.0^{\circ} \mathrm{s}^{-1}$ scan rate) examined in this study, a total of six cases of oriented plate-like crystals in clouds showed consistently strong angle-dependent effects and were selected for detailed study. Some of these observations were made in response to indications of oriented 
crystal effects by the PDL or polarization ruby lidar at FARS. Although this scanning dataset is too small to draw conclusions on the overall frequency of oriented ice crystal effects in cirrus clouds, more climatologically- representative results can be found in Sassen and Benson (2001).

The detailed FARS case study of 11 January 1999 will first be illustrated, before the analysis is applied to the entire dataset. The rare case featuring column crystals (Sassen and Takano 2000) will also be presented to highlight the different observations resulting from particle shape and fall variations.

\section{The 11 January 1999 Case Study}

\section{a. Lidar observations}

Between 2122 and 2330 UTC on 11 January 1999, a cirrus cloud located between 7.0 and $8.6 \mathrm{~km}$ (above mean sea level, MSL) was observed by the scanning lidar at the FARS location. The observations at the zenith angle tend to show $\delta<\sim 0.15$, while $\sim 0.45$ is measured in the surrounding cloud (Fig. 1a). In the zenith column between 7.0 and $7.5 \mathrm{~km}$, much lower values of $<0.05$ are indicated. As altitude increases above $7.5 \mathrm{~km}$, this minimum becomes less marked and becomes narrower, but is still visible up to $\sim 8.0 \mathrm{~km}$. Conversely, the relevant backscattered intensity (Fig. 1b) shows a marked increase in the same central region.

Observed values of $\delta$ in the horizontal cloud layer between 7.1 and $7.2 \mathrm{~km}$ are plotted in Fig. $2 \mathrm{a}$ (top) as a function of the lidar angle from the zenith $\theta$ (with $\theta=0^{\circ}$ being the zenith direction). Outside the central region $\left(\theta>4.0^{\circ}\right), \delta$ is gathered around $\sim 0.3$. Closer to the zenith, the depolarization ratio depends strongly on $\theta$, with many data points decreasing to near zero in the 
zenith angle. Values of $\delta$ show a high variability when viewed at these small spatial scales due to the effects of signal noise and the small cloud volumes sensed. This variability can be reduced by averaging the parallel and perpendicular lidar signals before computation of the ratio, although by averaging over too large scales most of the microphysical meaning of the depolarization ratio is lost, so this should be done with great care.

As also apparent in Fig. 1b, the backscatter intensity follows the opposite behavior, with a marked maximum in the zenith direction (Fig. 2b). Compared to lidar depolarization, these values are less scattered and the central peaked region is noticeably more narrow. This last difference can be explained by looking at how both values (depolarization ratio and backscattered intensities) depend on the number of horizontally-oriented crystals with respect to the entire population of crystals. Linear depolarization ratio in presence of horizontally-oriented particles can be written as

$$
\delta=\delta_{r} /\left[1+\left(N_{h} / N_{r}\right)\left(\beta_{h} / \beta_{r}\right)\right]
$$

where $\delta_{r}$ refers to the linear depolarization ratio for randomly-oriented particles, and $N$ and $\beta$ are the relative concentrations and backscattering coefficients for horizontally $(h)$ and randomly (r) oriented particles (Sassen and Benson, 2001). As Eq. 1 was initially developed for zenithpointing lidar, when considering a scanning lidar the $h$ subscript needs to be extended to include all planar particles with their hexagonal faces orthogonal to the lidar beam direction. Using $\delta_{r}=0.33$ for randomly oriented particles, $\delta=0.06$ at zenith (as Fig. 2a shows) and $\beta_{h} / \beta_{r}=360: 1$, as reported by Sassen (1977), in Eq. 1 leads to $N_{r} / N_{h}=80: 1$ at zenith.

Using the same notations, the backscattered intensity can be written as :

$$
\beta=\left[\beta_{h} / \beta_{r}\right] /\left[1+N_{r} / N_{h}\right]
$$


As generally $N_{r} / N_{h} \gg 1$, and $\beta_{h} / \beta_{r}$ is a constant, it follows that $N_{h} / N_{r}$ is directly proportional to $\beta$. As $N_{h} / N_{r}$ describes particles with hexagonal faces orthogonal to the lidar beam direction, basic geometry shows that the angular half-width of this ratio, and therefore of $\beta$, should be linked to the average deviation angle of horizontally-oriented crystals. This is coherent with observations, that show the angular half-width to be smaller for $\beta$ (correlated with the average deviation angle, Fig. 2b), than for $\delta$ (correlated with the maximum deviation angle, Fig. 2a). Normalizing the backscattered intensity to $\beta=1$ at zenith, Figure 3 shows that backscattered intensity decreases much faster than depolarization ratio increases. A noticeable impact on backscattered intensity requires a much lower ratio $N_{r} / N_{h}$ (hence an incidence angle closer to zenith) than for depolarization ratio, leading to a narrower peak on angle-dependent observations.

However, the backscattered intensity, unlike the depolarization ratio, is attenuated by the cloud optical depth. Considering oriented crystals at a given altitude, the length of the laser path will increase with the lidar incidence angle $\theta$, and more particles will contribute to laser attenuation. Due to the inhomogenous distribution of particle concentrations in the cloud, this change in attenuation is difficult to quantify. The depolarization ratio is not affected by this problem, and seems more adapted to the current method of analysis.

\section{b. Analytical model and retrieval technique}

The lidar depolarization ratio display of Fig. 2a suggests that the angular evolution of $\delta$ in a given cloud height layer can be described with three parameters :

- The linear depolarization ratio value in the zenith direction $\delta_{\text {zen }}$, 
- The linear depolarization ratio value outside the central region $\delta_{\text {off, }}$

- The angular half-width of the central peak $\sigma$.

In agreement with earlier studies, our observations strongly suggest a Gaussian function, so by taking into account the three parameters $\delta_{\text {zen }}, \delta_{\text {off }}$ and $\sigma$, a possible analytical model for the depolarization ratio is given by the function :

$$
\delta_{\text {sim }}(\theta)=\delta_{\text {off }}-\exp \left(\frac{\theta^{2}}{2 \sigma^{2}}\right)\left(\delta_{o f f}-\delta_{z e n}\right)
$$

It is important to note that in this model the Gaussian function standard deviation $\sigma$ is also the angular half-width of the central peak and marks the inflexion point of the evolution of angular $\delta$ values. As the observed inflexion point is linked to the angular deviation of crystals, retrieving $\sigma$ using this model will directly quantify the maximum deviation of crystals from the horizontal plane in the each cloud. A near-perfect horizontal orientation of plate crystals will produce a narrow peak of $\delta$ in the zenith direction and a low value of $\sigma$.

To fit the simulated function $\delta_{\operatorname{sim}}(\theta)$ to actual observations, only complete lidar scans are considered. In each scan, $\delta$ is considered in horizontal layers defined by consecutive altitude bins (the data shown in Fig. 2 is a good example). First approximations for $\delta_{\text {zen }}$ and $\delta_{\text {off }}$ are obtained by considering near-zenith $\left(\theta<0.5^{\circ}\right)$ and off-zenith $\left(\theta>5.0^{\circ}\right)$ observations in each altitude range. These values are used as a starting point for a Levenberg-Marquardt least-square minimization fitting technique. Stopping the process after 700 iterations was found to provide stable results. Variations of 0.2 around the mean values were allowed for $\delta_{\text {zen }}$ and $\delta_{\text {off }}$, while a variation range 
betwen 0 and 10 is allowed for $\sigma$. For the retrieved parameters to be considered as part as an oriented crystal area, three conditions must be fulfilled.

\section{i. High off-zenith depolarization ratio}

To ensure the presence of an ice cloud in the considered horizontal layer, high values of offzenith depolarization ratio $\delta_{\text {off }}$ are required (typically, $\delta_{\text {off }}>0.05$ was found to be sufficient). For the 11 January 1999 case, $\delta_{\text {off }}($ Fig. 4) slowly increases from 0.3 at the cloud base $(6.9 \mathrm{~km})$ to 0.38 at the cloud top $(8.6 \mathrm{~km})$. The higher value above $8.6 \mathrm{~km}$ implies a difference in ice particle shapes.

ii. Significantly lower zenith depolarization ratio

High values of $\delta_{\text {off }}$ and low values of zenith depolarization ratio $\delta_{\text {zen }}$ are a strong indication of oriented ice crystals. However, variations in $\delta$ with the incidence angle could also come from other spatial microphysical changes in the cloud. To ensure the presence of oriented ice crystals, a significant decrease in depolarization ratio between off-zenith and zenith is required (typically, a $10 \%$ decrease was found to be sufficient). For the 11 January 1999 case, the vertical profile of $\delta_{\text {zen }}$ (dashed line in Fig. 4) stays below 0.2 between $6.9 \mathrm{~km}$ (the cloud base) and $7.5 \mathrm{~km}$, i.e. a more than $30 \%$ decrease from the off-zenith values. For altitudes above $7.5 \mathrm{~km}, \delta_{\text {zen }}$ is higher but still low enough to warrant the detection of oriented crystals. This increase in $\delta_{\text {zen }}$ could be explained either by a lower relative concentration of horizontally-oriented crystals (Sassen and Benson 2001), or by a change in orientation behavior due to different particle shapes or sizes. As the horizontal extent for the present case is small enough $(<1.0 \mathrm{~km})$ to consider the particle shape to be horizontally homogeneous, the increase in $\delta_{\text {off }}$ implies a change in particle shape. 
iii. Low deviation from the Gaussian Model

Even when conditions (i) (high values of $\delta_{\text {off }}$ ) and (ii) (low values of $\delta_{\text {zen }}$ ) are fulfilled, the fitting technique is sometimes not applicable : horizontal in-cloud variations of particle shape can also lead to such conditions resembling the signature of horizontally oriented plate crystals. To filter out these situations, the average difference is computed between the gaussian model and the actual observations. If this difference is higher than $20 \%$, the retrievals are not considered to be representative of oriented crystals.

These remarks also hold true when considering observations of backscattered intensities (Fig. 2b). Taking into account that intensities are increasing in the zenith region instead of decreasing, the same technique can be applied.

\section{c. Model retrieved parameters}

As an example, fitting the function in Eq. 3 to 11 January 1999 observations for altitude range 7.1 to $7.2 \mathrm{~km}$ in Fig. 2 leads to retrieved parameters $\delta_{\text {zen }}=0.09, \delta_{\text {off }}=0.32$ and $\sigma=1.96^{\circ}$. The resulting function (bold line over the observations in Fig. 2a) follows very closely the angular evolution of observations.

This retrieval technique was applied to all horizontal layers for each consecutive lidar scan of the 11 January 1999 case. Retrieved angular widths plotted as a function of altitude (symbols in Fig. 5a, averaged in full line) shows $\sigma \sim 1.4^{\circ}$ at cloud bottom (near $7.0 \mathrm{~km}$ ), decreasing to as low as $\sigma \sim 0.4^{\circ}$ at mid-cloud $(7.5 \mathrm{~km})$, and increasing again up to $\sigma \sim 1^{\circ}$ at higher levels. The absence of valid retrievals above $8.1 \mathrm{~km}$ is due to the uncertainties becoming too large to ensure a good fit between the observations and the analytical model. These results reflect closely the vertical changes in the vertical column of low $\delta$ shown in Fig. 1. 
A similar analysis was conducted on the corresponding observations of backscattered intensities. For the horizontal cloud layer shown in Fig. $2 \mathrm{~b}$, a value of $\sigma=0.90^{\circ}$ was found for the angular half-width. The vertical profile of retrieved angular widths (Fig. 5b) shows that results are only retrieved in the $7.0-7.5 \mathrm{~km}$ altitude range, and that successful retrievals are fewer than when studying the linear depolarization ratio. The disappearance of the central peak for higher altitude is also apparent in the scanning image (Fig. 1b). The fitting technique is overall less successful than with depolarization ratio. However, it should be noted that in this limited altitude range, the peaks of high intensities in the zenith area are narrower (generally $\sigma<1^{\circ}$ ) than their depolarization counterparts $\left(0.7^{\circ}<\sigma<1.4^{\circ}\right.$ in Fig. $\left.5 \mathrm{a}\right)$.

\section{A singular case of Parry-oriented columns}

On 16 November 1998, a cirrus producing an upper Parry Arc was probed by the scanning PDL for more than 6 minutes, revealing unusual observations of angle-dependent $\delta$ (Sassen and Takano 2000). The example in Fig. 6 shows an off-zenith depolarization ratio $0.35<\delta_{\text {off }}<0.45$, with a much lower $\delta \sim 0.2$ in the zenith direction, following the plate crystal behavior. However, for a small tilt angles from the zenith, extremely high values of depolarization ratio $(\delta>0.8)$ are observed, which is clearly different from the behavior of oriented plate-like crystals showed in case 1 (Sect. 4).

Values of $\delta$ in the horizontal cloud layer between 8.1 and $8.2 \mathrm{~km}$, plotted as a function of the lidar angle from zenith $\theta$ (Fig. 7), reveal a slight asymmetry (the minimum $\delta$ is found at $\theta$ $=+0.5^{\circ}$ ), which can be attributed to a small error in the level of the PDL system. Taking this 
offset into account, the maximum values of $\delta \sim 1.2$ are found at incidence angles $1.5^{\circ}$ off the zenith. This rare angular evolution of $\delta$ was explained in Sassen and Takono (2000) by the presence of column crystals with a pair of prism faces parallel to the ground (the so-called Parryorientation). To produce the observed displays, these crystals should bear an almost perfect horizontal orientation (deviation from horizontal below $0.5^{\circ}$ ). This case highlights the strong sensitivity of scanning observations of depolarization ratios to the crystal fall behavior, and shows that angular-dependent observations are a powerful way to discriminate between particle shapes.

This unusual behavior was only observed once in the cases analyzed for the present study, and as such is an exception. Cases presented in the rest of this paper follow the behavior described earlier, and are classified as plate-like crystals.

\section{Retrieval of deviation angles for additional cases}

The properties of six cases of oriented crystals observed in cirrus and midlevel clouds by scanning lidar are summarized in Table 1, including the thickness and temperature ranges of oriented crystal layers. Oriented crystals can be observed for time periods as long as 25 minutes. Example scans for cases 2 to 6, similar to the one shown in Fig. 1 for case 1, are given in Fig. 8. The fitting technique using Eq. 3 was applied, with the three criterias (Sect. 4.b) being fulfilled in $13.5 \%$ of all horizontal layers. In each case, the three parameters, $\delta_{\text {zen }}, \delta_{\text {off }}$ and $\sigma$ were retrieved. However, the layers of horizontally oriented crystals do not share the same altitudes. To allow for the study of the vertical evolution of retrieved parameters, the observations are analyzed as a function of therelative penetration depth into the layer of oriented crystals $(0 \%$ meaning layer 
bottom and $100 \%$ layer top). In all cases, ice clouds continued to be probed above the oriented crystals layer.

The particle orientation behavior mostly depends on the particle shape (Klett 1995). In absence of a preferential orientation of crystals, the lidar depolarization ratio is indicated to convey meaningful information about the shape of crystals probed by polarized laser light (Sassen 1991). Thus $\delta_{\text {off }}$ can reveal possible differences in crystal shape between the studied cases. Plotting $\delta_{\text {off }}$ as a function of layer penetration (symbols in Fig. 9, averaged profiles in thick lines) seperates the cases into two groups centered around $\delta_{\text {off }} \sim 0.25$ (cases 3, 4 and 5, diamonds and full line in Fig. 9) and $\delta_{\text {off }} \sim 0.35$ (cases 1, 2 and 6, crosses and dashes line in Fig. 9). Table 1 shows that $\mathrm{T}>-20^{\circ} \mathrm{C}$ for oriented crystals in the first group (warm clouds), and $\mathrm{T}<-30^{\circ} \mathrm{C}$ for the second group (cold clouds). This seperation can be explained by the strong influence of temperature on the crystal growth process, and therefore on their shape, which controls the orientation behavior. In the following sections, retrieved parameters will be seperated into these two groups, to highlight possible different orientation behavior modes based on particle shape. The fact that no oriented crystals are found below $-40^{\circ} \mathrm{C}$ is a consequence of the limited dataset, as crystal orientation is present at all temperatures (Thomas et al, 1990).

\section{a. Vertical profiles of angular widths}

Angular widths for the warmer clouds (Fig. 10a) are very scattered with altitude. However, the average profile for all cases (bold line) stays between $1.5^{\circ}$ and $2.1^{\circ}$ for a penetration depth below $75 \%$. For higher penetration depths, $\sigma$ then decreases to reach $1.1^{\circ}$ at relative cloud top. Angular widths retrieved for the colder clouds (Fig. 10b) follow a behavior similar to the 11 January 1999 
case (Fig. 5). The average profile for all cases (bold line) shows a maximum angular width ( $\sigma \sim$ $\left.1.3^{\circ}\right)$ located at the layer bottom. We believe that when oriented crystal effects are present near cloud base, the angular widths of the near-zenith depolarization trough (and the backscattering peak) characteristically increase as a result of the effects of ice crystal evaporation, which reduces ice crystal size and thus increases the fall instability (Sassen 1980). The angular width then decreases with increasing altitude, with a minimum value $\left(\sigma \sim 0.6^{\circ}\right)$ reached at $40 \%$ penetration depth. Finally $\sigma$ increases again, with a second maximum $\sigma \sim 0.8^{\circ}$ observed around $80 \%$ penetration.

\section{b. Angular frequencies}

Overall, valid angular half-widths highlighting the presence of oriented crystals were retrieved in $27.6 \%$ of the cloud layer bins for the 6 selected case studies. This frequency should not be extended to all ice clouds, however, because the dataset was selected to favour oriented crystal observations, and the analysis method used here rejects weak orientation effects. It shows, though, that when oriented crystals are present, their coverage can extend on average to a third of the cloud layer.

In the studied warmer cloud cases, oriented crystals were detected in $22.5 \%$ of the probed altitude ranges. Retrieved angles for these clouds (Fig. 11a) follow a rather smooth distribution, with maximum frequencies for half-width angles $1.75^{\circ}<\sigma<2.25^{\circ}$, and $54 \%$ of them between $1.0^{\circ}$ and $3.0^{\circ}$. Frequencies decrease as angles move away from this range : $18 \%$ of angles are below $1.0^{\circ}$, with a minimum $6 \%$ reached for angles below $0.25^{\circ} ; 28 \%$ of angles are above $3.0^{\circ}$, with frequencies decreasing slowly to $4 \%$ for angles $>4.75^{\circ}$. 
In the studied cases of colder clouds, oriented crystals were detected in $35.8 \%$ of the probed altitude ranges. Retrieved half-width angles for these clouds (Fig. 11b) follow a more narrow distribution, centered on lower angles ranging from $0.75^{\circ}<\sigma<1.25^{\circ}$. Only $18.5 \%$ of angles are above $1.75^{\circ}$, with that number falling under $7 \%$ above $3.0^{\circ}$. Small angles are dominant, with $81.5 \%$ of angles below $1.75^{\circ}$, compared to $36.5 \%$ for warmer clouds (Fig. 11a).

\section{c. Angles retrieved from analysis of backscattered power}

The angular variations of observed backscattered intensities were also fitted to the model in Eq. 3 for all cases. However, as noted earlier, results from the analysis of backscattered intensities often have higher uncertainties than when studying depolarization ratios. For cases when angle retrieval was possible using $\delta$ scans, only $5 \%$ of the cases analyzed using backscattered intensities gave confident results. For those cases, the retrieved angular deviations $\sigma_{\text {pow }}$ are plotted in Fig. 12 as a function of the corresponding angular deviation $\sigma$ retrieved from the $\delta$ analysis. When the depolarization ratio analysis leads to angular deviations between 0.25 and 3.0 ${ }^{\circ}$, the associated $\sigma_{\text {pow }}$ lies between 0.2 and $2.0^{\circ}$. Least-square fitting on a second-order polynomial (dotted line) shows that for an increase from 0.25 to $0.3^{\circ}$ in $\sigma, \sigma_{\text {pow }}$ slowly increases from $0.2^{\circ}$ and reaches saturation around $2.0^{\circ}$. As the half-widths from backscattered power are linked to the average deviation angle (Sect. 4a), this shows that the maximum deviation angle increases faster than the average deviation angle. As the variability of angles retrieved from backscattered intensities seems to be lower than from analysis of depolarization ratios, this suggests the angular evolution of the lidar depolarization ratio is more sensitive to changes in microphysical properties, and is better suited for studies of crystal orientation. 


\section{Discussion and Conclusions}

A technique aimed at estimating the maximum deviation angle of horizontally-oriented plate crystals was presented, fitting the variation of depolarization ratio with the lidar incidence angle to a Gaussian model. The model reproduces observations of $\delta$ for plate-like particle cases. A very rare case of oriented columns exhibits a strongly contrasting behavior, which prevents confusion between the two crystal shapes.

Planar crystals in warm $\left(>\sim-20^{\circ} \mathrm{C}\right)$ midlevel clouds show overall a higher half-width angle, centered on an average $2.0^{\circ}$ and distributed smoothly between 0 and $5.0^{\circ}$ (Fig. 11a). For crystals in cold $\left(<\sim-30^{\circ} \mathrm{C}\right)$ cirrus clouds, more than half of the retrieved angles below $1.0^{\circ}$ (Fig. 11b). As the half-width is related to the maximum deviation angle, crystals appear more closely oriented with the horizontal planes in cold clouds. This difference must come from different particle shapes, as suggested by the difference in $\delta_{\text {off }}$ between cold and warm clouds (Fig. 9). The most plausible explanation is that the differences in temperature have favored the growth of large and more widely fluttering dendritic ice crystals at $>\sim-20^{\circ} \mathrm{C}$, although the $\delta$ values for dendrites have not yet been estimated from theory. An analog exists, however, in terms of observations : the sun pillar optical phenomenon, which is caused by the reflections from fluttering ice crystals off the setting/rising sun. The angular height of the pillar increases as the crystal wobble angle from the horizontal plane increases, and extended observations show that proper sun pillars are rare in cirrus clouds, but often spectacular in the ice crystals falling below mixed phase clouds at warmer temperatures (Sassen et al. 2003). Similarly, the tendency for the deviation angles to increase significantly at cloud base also reflects wider fluttering motions, which in this case seem to be 
due to the effects of crystal evaporation that decrease crystal size and fall atitude stability. These different orientation behaviors could also be explained by atmospheric dynamics : more laminar air motions in higher, colder clouds, would lead to almost-horizontal orientations, while more turbulent motions in lower, warmer clouds, unable to destroy the orientation itself (Cho et al. 1981), would nethertheless lead to an increase in crystal deviation from the horizontal plane.

Among the six studied cases, crystals in cold clouds are more closely aligned with the horizontal plane, and their angular half-width is smaller by $1.0^{\circ}$ than in warm clouds, so the maximum deviation angle should follow the same trend. Moreover, the relative frequency of oriented crystals is higher in cold $(\sim 35 \%)$ clouds than warm $(\sim 22 \%)$ clouds. If this behavior holds true for more robust sample sizes, it may have noticeable radiative consequences. Horizontally oriented particles increase the hemispherical albedo effect of a given cirrus cloud, and the more closely aligned are the particles, the higher the increase. The impact of particle alignment on the radiative properties has to be quantified, but if results from the present study are representative of most ice clouds, oriented crystals would influence more the radiative balance of cirrus clouds than other ice clouds. As changes in high cloud albedo directly impact the transfer of radiation through the troposphere at visible wavelengths, this phenomenon could have important consequences for the global radiative budget. To estimate these effects, large-scale studies of crystal orientation in cirrus clouds are required. This could be possible through application of similar analysis to multi-angle satellite observations. For instance, polarized radiances from the POLDER instrument have been shown to contain valuable information on oriented particles (Chepfer et al, 1999; Noel and Chepfer, 2004). However, as was shown in the present paper, scanning lidar not only allows an analysis of the crystal orientation, but also 
provides ways to correlate the retrieved properties with their synoptic and microphysical context. The presence of such an instrument on a spaceborne platform (Winker et al. 2003), with a smaller scanning angle $\left(\right.$ e.g. $\left.1^{\circ}\right)$ to compensate for the high altitude, would greatly improve the current knowledge of cirrus clouds microphysics in general.

Acknowledgements. This research was supported in part by NASA-Langley Research Center, under contract NAS1-02058. The FARS data used in this study has been collected and analyzed under support from NFS grants ATM-9528287 and ATM-0296190. The PDL system was developed under DOE grant DEFG-0394ER747 from the Atmospheric Measurement Program. The authors would like to thank Dave M. Winker for his support, and the reviewers for their useful comments. 


\section{References}

Chepfer, H., G. Brogniez, P. Goloub, F. M. Breon and P. H. Flamant, 1999: Observations of horizontally oriented ice crystals in cirrus clouds with POLDER-1/ADEOS-1. J. of Quant. Spectrosc. Radiat. Transfer, 63, 521-543.

Cho, H. R., J. V. Iribarne and W. G. Richards, 1981: On the orientation of ice crystals in a cumulonimbus cloud. J. Atmos. Sci., 34, 1111-1114.

Klett, J. D., 1995: Orientation model for particles in turbulence. J. Atm. Sci., 52, 2276-2285.

Liou, K. N., 1986: Influence of cirrus clouds on weather and climate processes: A global perspective. J. Geophys. Res., 103, 1799-1805.

Liou, K. N. and H. Lahore, 1974: Laser sensing of cloud composition : A backscattered depolarization technique. J. Atmos. Sci., 13, 257-263.

Magono, C., 1953: On the growth of snow flakes and graupel. Sci. Rep. Yokohama Natl Univ., Ser. 1, 2: 18-40.

Masuda, K. and H. Ishimoto, 2004: Influence of particle orientation on retrieving cirrus cloud properties by use of total and polarized reflectances from satellite measurements. J. Quant. Spectrosc. Rad. Trans., 85, 183-193.

Mishchenko, M. I., D. J. Wielaard, and B. E. Carlson, 1997: T-matrix computations of zenith-enhanced lidar backscatter from horizontally oriented ice plates. Geophys. Res. Let., 24, 771-774.

Noel, V. and H. Chepfer, 2004: Study of ice crystal orientation in cirrus clouds based on satellite polarized radiance measurements. J. Atmos. Sci., accepted, UPDATE??? 
Noel, V., G. Roy, L. Bissonnette, H. Chepfer and P. H. Flamant, 2002: Analysis of ice clouds lidar measurements at multiple incidence angles. Geophys. Res. Let., 29, 52-1 $52-4$.

Ono, A., 1969: The shape and riming properties of ice crystals in natural clouds. J. Atmos. Sci., 26, 138-147.

Pal, S. R., and A. I. Carswell, 1973 Polarization properties of lidar backscattering from clouds. Applied Optics, 12, 1530-1535.

Platt, C. M. R., 1978: Lidar backscatter from horizontal ice crystal plates. J. Appl. Meteor., $17,482-488$.

Platt, C. M. R., N. L. Abshire, and G. T. McNice, 1978: Some microphysical properties of an ice cloud from lidar observations of horizontally oriented crystals. J. Appl. Meteor., 17, 1220-1224.

Roy, G., and L. Bissonnette, 1999: Retrieval of droplet-size density distribution from multiple field-of-view cross-polarized lidar signal: theory and experimental validation. Applied Optics, 38, 5202-5211.

Sassen, K., 1977: Ice crystal habit discrimination with the optical backscatter depolarization technique. J. Appl. Meteor., 16 425-431.

Sassen, K., 1980: Remote sensing of planar ice crystals fall attitude. J. Meteor. Soc. Japan, 58, 422-433.

Sassen, K., 1987: Polarization and brewster angle properties of light pillars. J. Opt. Soc. Am., 4, 570-580. 
Sassen, K., 1991: The polarization lidar technique for cloud research: A review and current assessment. Bull. Am. Meteor. Soc., 71, 1848-1866.

Sassen, K., 1994: Advances in polarization diversity lidar for cloud remote sensing. Proc. IEEE, 82,1907-1914.

Sassen, K., and Y. Takano, 2000: Parry arc: A polarization lidar, ray-tracing, and aircraft case study. Applied Optics, 39, 6738-6475.

Sassen, K., and S. Benson, 2001: A midlatitude cirrus cloud climatology from the facility for atmospheric remote sensing. Part II: Microphysical properties derived from lidar depolarization. J. Atmos. Sci., 58, 2103-2111.

Sassen, K., J. Zhu, and S. Benson, 2003: A midlatitude cirrus cloud climatology from the Facility for Atmospheric Remote Sensing: IV. Optical displays. Appl. Opt., 42, 332341.

Schotland, R. M, K. Sassen,. and R. J. Stone, 1971: Observations by lidar of linear depolarization ratios by hydrometeors. J. Appl. Met., 10, 1011-1017.

Stephens, G. L., S.-C. Tsay, P. W. Stackhouse, and P. J. Flatau, 1990: The relevance of the microphysical and radiative properties of cirrus clouds to climate and climate feedback. J. Atmos. Sci., 47, 1742-1753.

Takano, Y., and K. N. Liou, 1989: Solar radiative transfer in cirrus clouds. Part 2: Theory and computation of multiple scattering in an anisotropic medium. J. Atmos. Sci., 46, $20-36$.

Takano, Y., K. N. Liou, and P. Minnis, 1992: The effect of small ice crystals on cirrus infrared radiative properties. J. Atmos. Sci., 49, 1487-1493. 
Thomas, L., J. C. Cartwright and D. P. Wareing, 1990: Lidar observations of the horizontal orientation of ice clouds in cirrus clouds. Tellus, volume 42B, 211-216.

Winker, D. M., J. Pelon and M. P. McCormick, 2003: The CALIPSO Mission : Spaceborne Lidar for observations of Aerosols and Clouds. Proc. SPIE, volume 4893, 1-11.

Young, A. T., 1980: Revised depolarization corrections for atmospheric extinction. Applied Optics, vol. 19, 3427-3428. 


\section{Table Captions}

Table 1 : Properties of the layers of Oriented Crystals (OC) observed in cirrus clouds by scanning lidar in the studied cases. 


\begin{tabular}{lcccccrr}
\hline \hline Case & Date & $\begin{array}{c}\text { Time } \\
{[\mathrm{min}]}\end{array}$ & $\begin{array}{c}\text { Cloud altitude } \\
{[\mathrm{km}]}\end{array}$ & $\begin{array}{c}\text { OC Layer Thickness } \\
{[\mathrm{km}]}\end{array}$ & \multicolumn{2}{c}{ OC Freq. Top T } & Bottom $\mathrm{T}^{\circ}$ \\
\hline 1 & $1999-01-11$ & 12 & $6.9-8.6$ & 0.9 & 50.9 & -32.6 & -26.8 \\
2 & $1999-01-11$ & 8 & $6.3-8.3$ & 0.8 & 35.7 & -32.0 & -26.8 \\
3 & $2000-11-22$ & 25 & $4.8-6.4$ & 0.6 & 20.7 & -22.6 & -17.8 \\
4 & $2001-04-27$ & 12 & $4.2-6.5$ & 0.4 & 25.0 & -16.7 & -13.4 \\
5 & $2000-03-27$ & 17 & $4.0-5.0$ & 0.4 & 22.0 & -7.9 & -6.8 \\
6 & $1998-11-16$ & 12 & $7.4-10.0$ & 0.8 & 25.8 & -36.3 & -30.3 \\
\hline \hline
\end{tabular}

Table 1: 


\section{Figure Captions}

Figure 1. a. PDL scanning observations of linear depolarization ratio at $\pm 10^{\circ}$ zenith angle on 11 January 1999. b. Same as 'a' but for backscattered intensities.

Figure 2. a. Observations of linear depolarization ratio as a function of the lidar incidence angle between 7.1 and $7.2 \mathrm{~km}$. b. Same as 'a' but for backscattered intensities.

Figure 3. Model results showing the dependence of the linear depolarization ratio and backscattered intensity on the ratio of randomly to horizontally oriented plate ice crystals.

Figure 4. Profile of off-zenith linear depolarization ratio.

Figure 5. a. Retrieved half-width angles $\sigma$ for Gaussian functions fitted to depolarization ratios as a function of altitude. b. Same as 'a' but fitted to backscattered intensities.

Figure 6. Same as Fig. 1 but for the 16 November 1999 case.

Figure 7. Same as Fig. 2 but for 16 November 1999 case between 8.1 and $8.2 \mathrm{~km}$.

Figure 8. Same as Fig. 1 but for cases 2 to 6.

Figure 9. Profiles of off-zenith linear depolarization ratios for all cases.

Figure 10. a. Retrieved half-width angles $\sigma$ for gaussian functions fitted to depolarization ratios as a function of altitude, for warmer clouds. Dashed lines are averages for each single case, and the full line is the total average. b. Same as 'a' but for colder clouds.

Figure 11. a. Histogram of retrieved half-width angles $\sigma$ for warmer clouds. b. Same as 'a' but for colder clouds.

Figure 12. Deviation angles retrieved from analysis of backscattered intensities plotted as a function of the corresponding angles retrieved from analysis of linear depolarization ratio. 

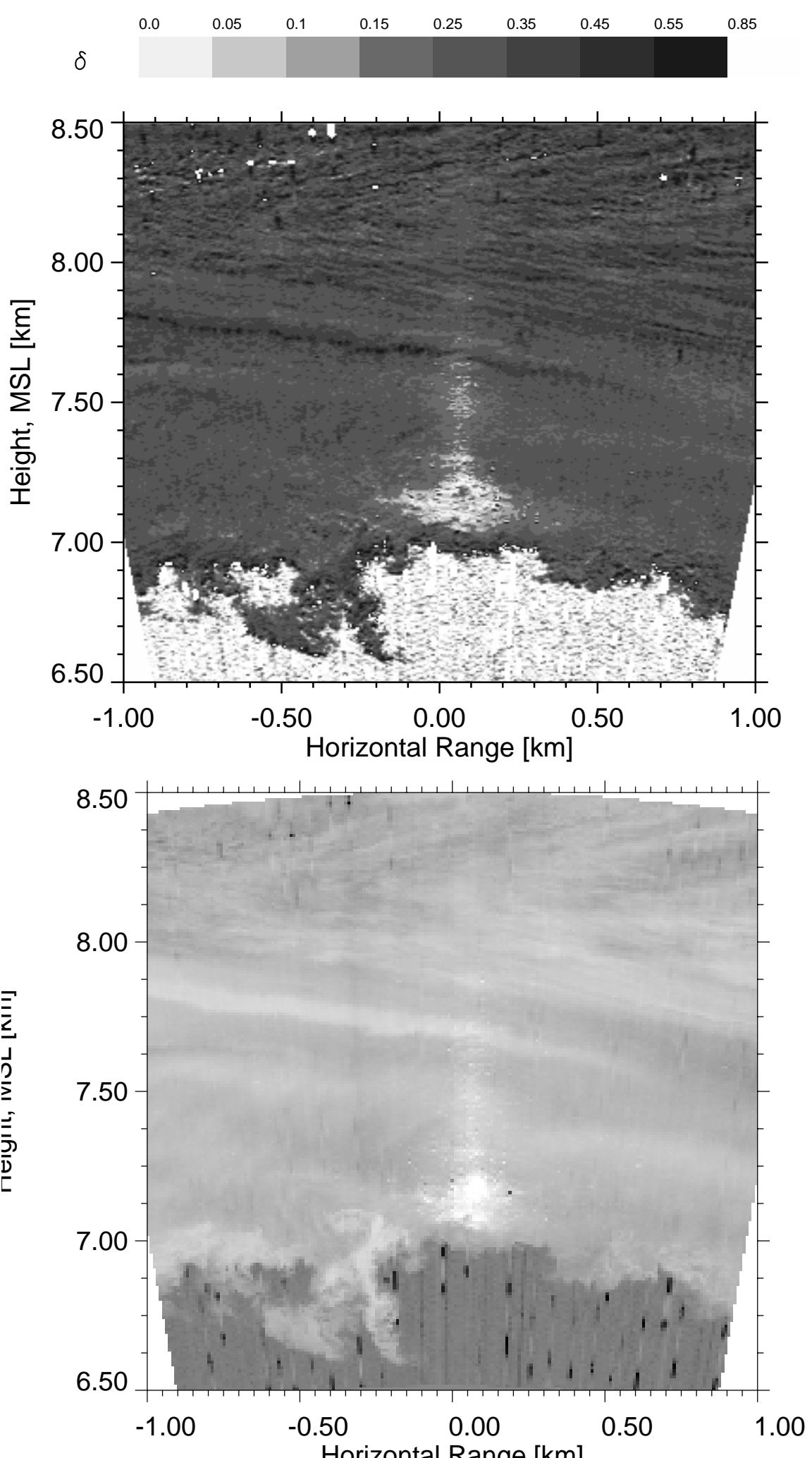

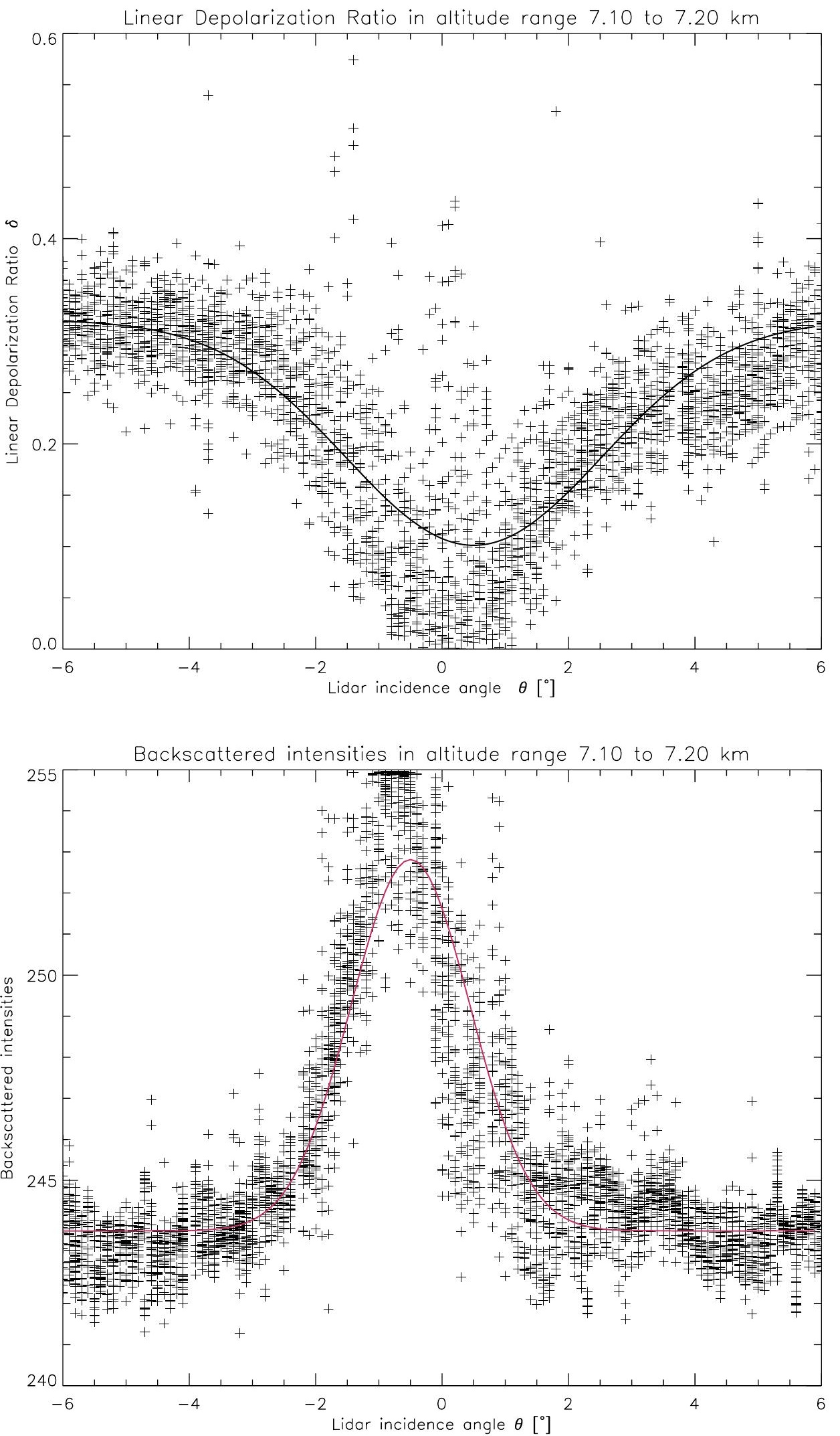


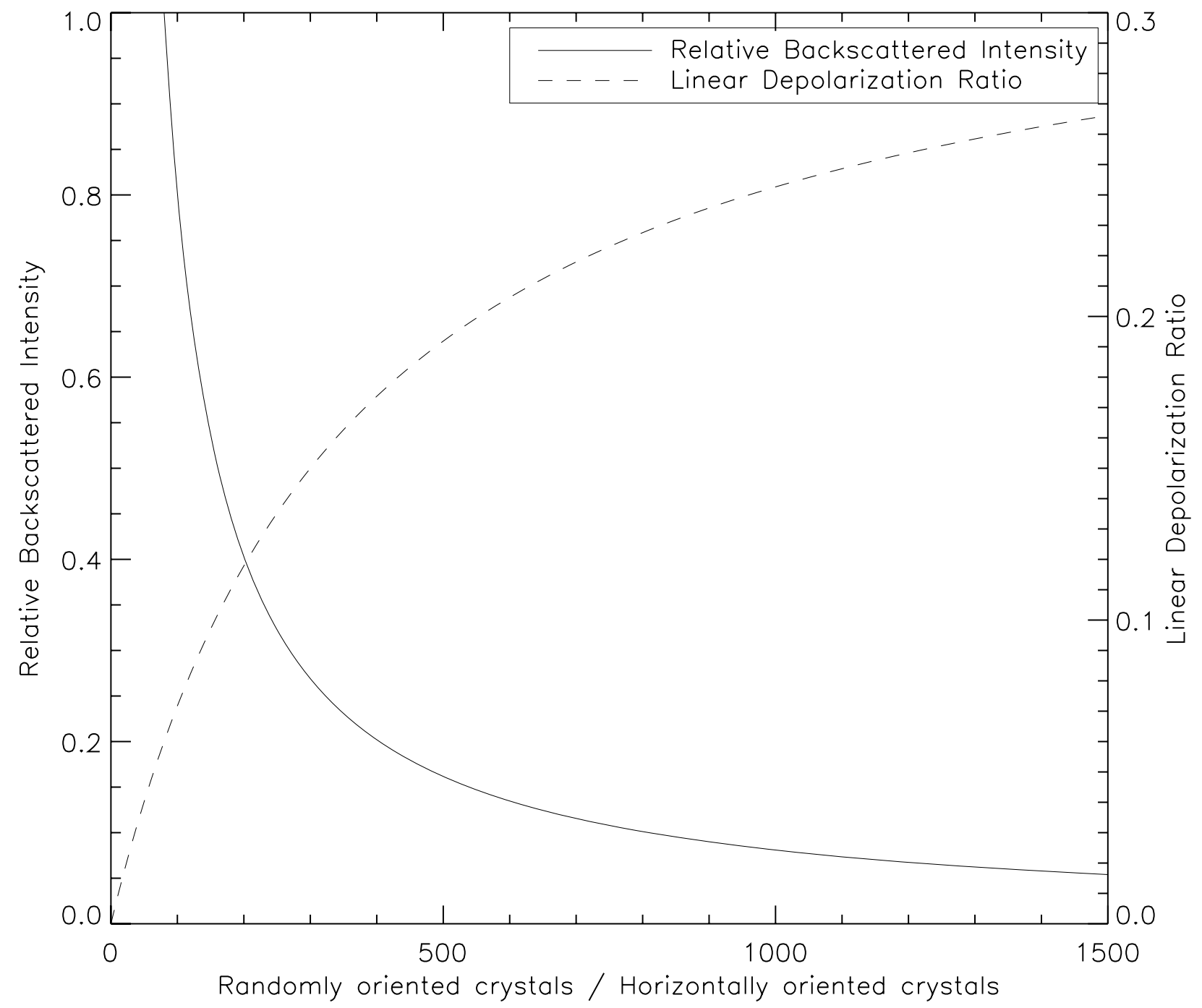




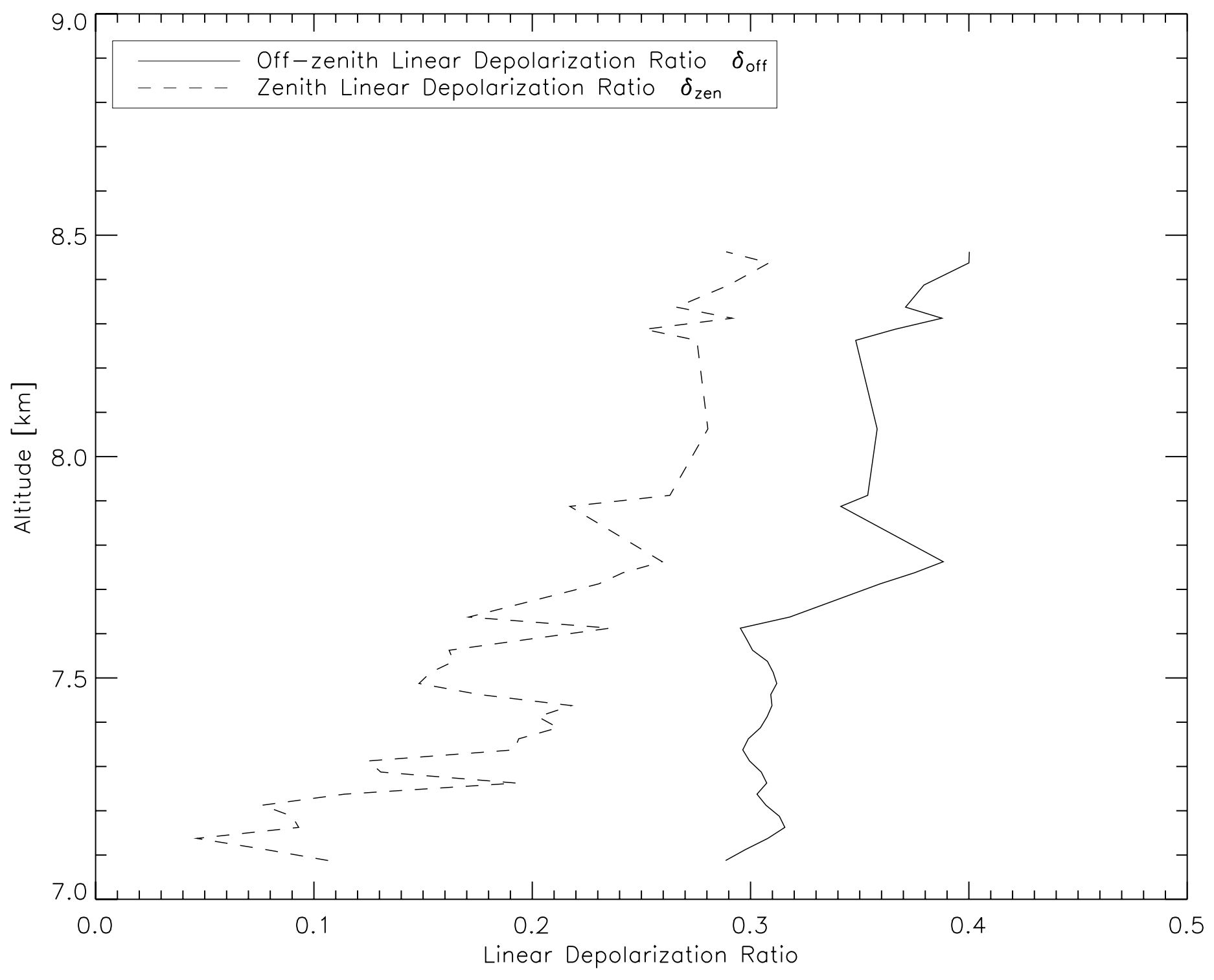



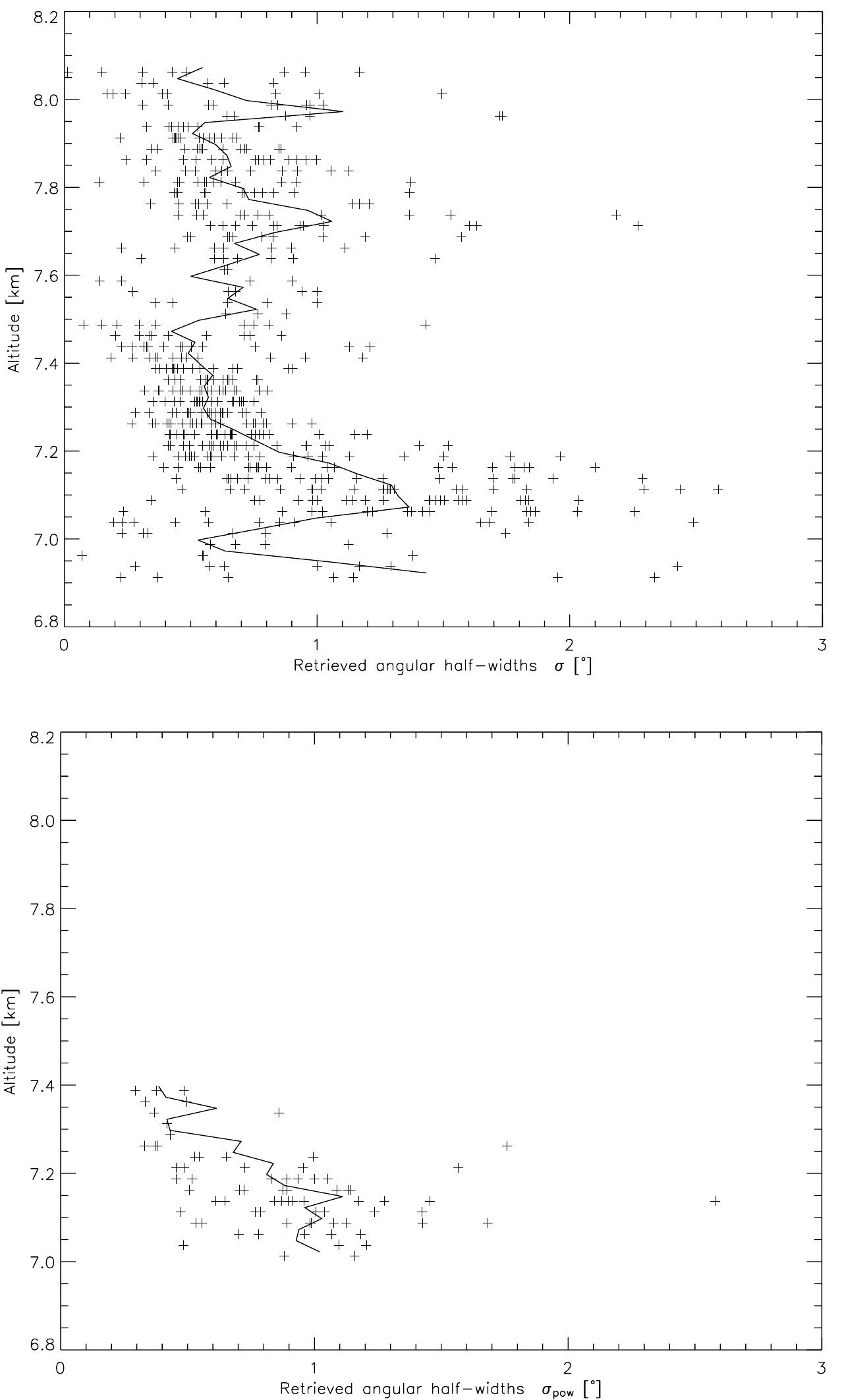


$\begin{array}{lllllllll}0.0 & 0.05 & 0.1 & 0.15 & 0.25 & 0.35 & 0.45 & 0.55 & 0.85\end{array}$

$\delta$

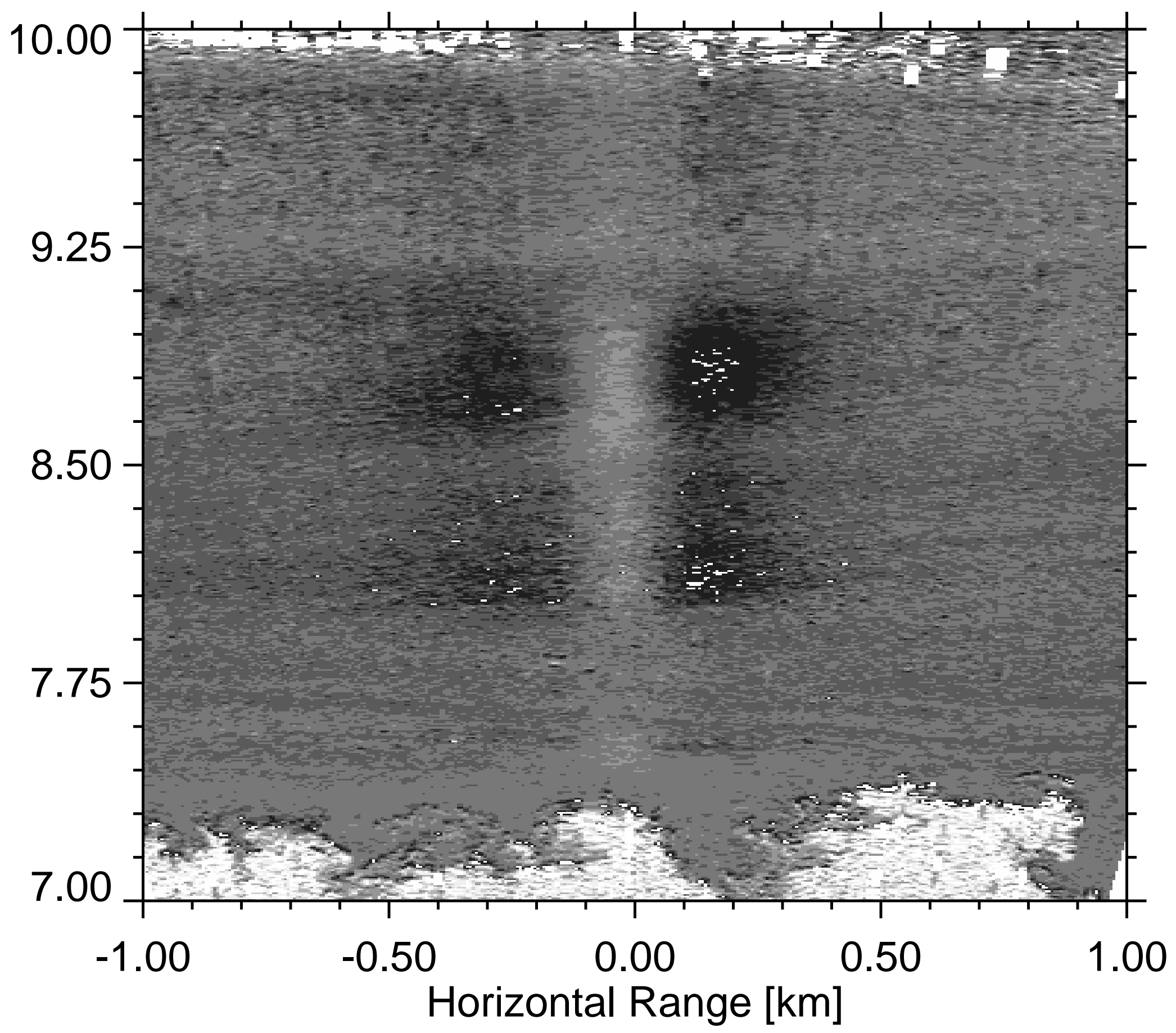


Linear Depolarization ratio in altitude range 8.10 to $8.20[\mathrm{~km}]$

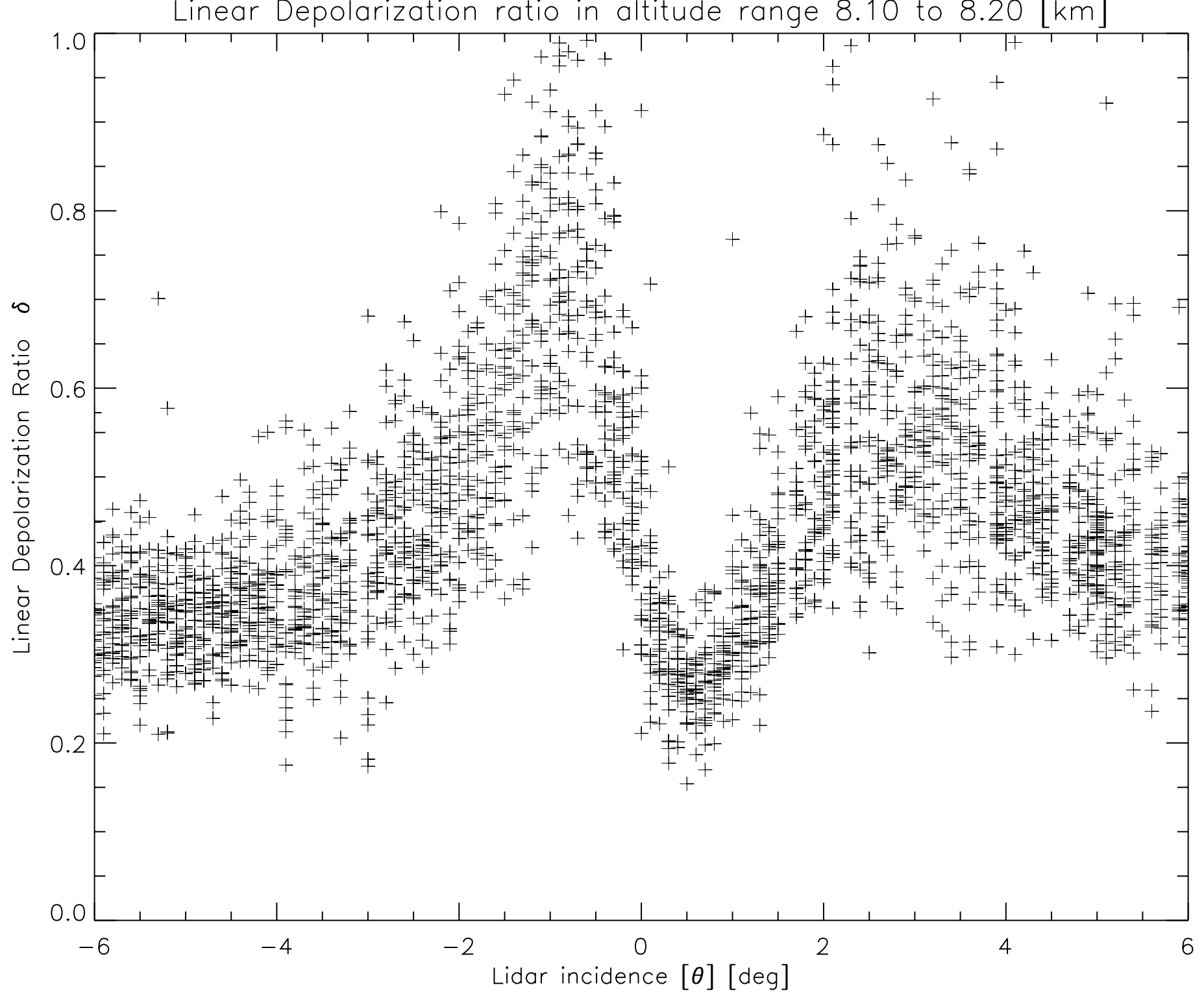



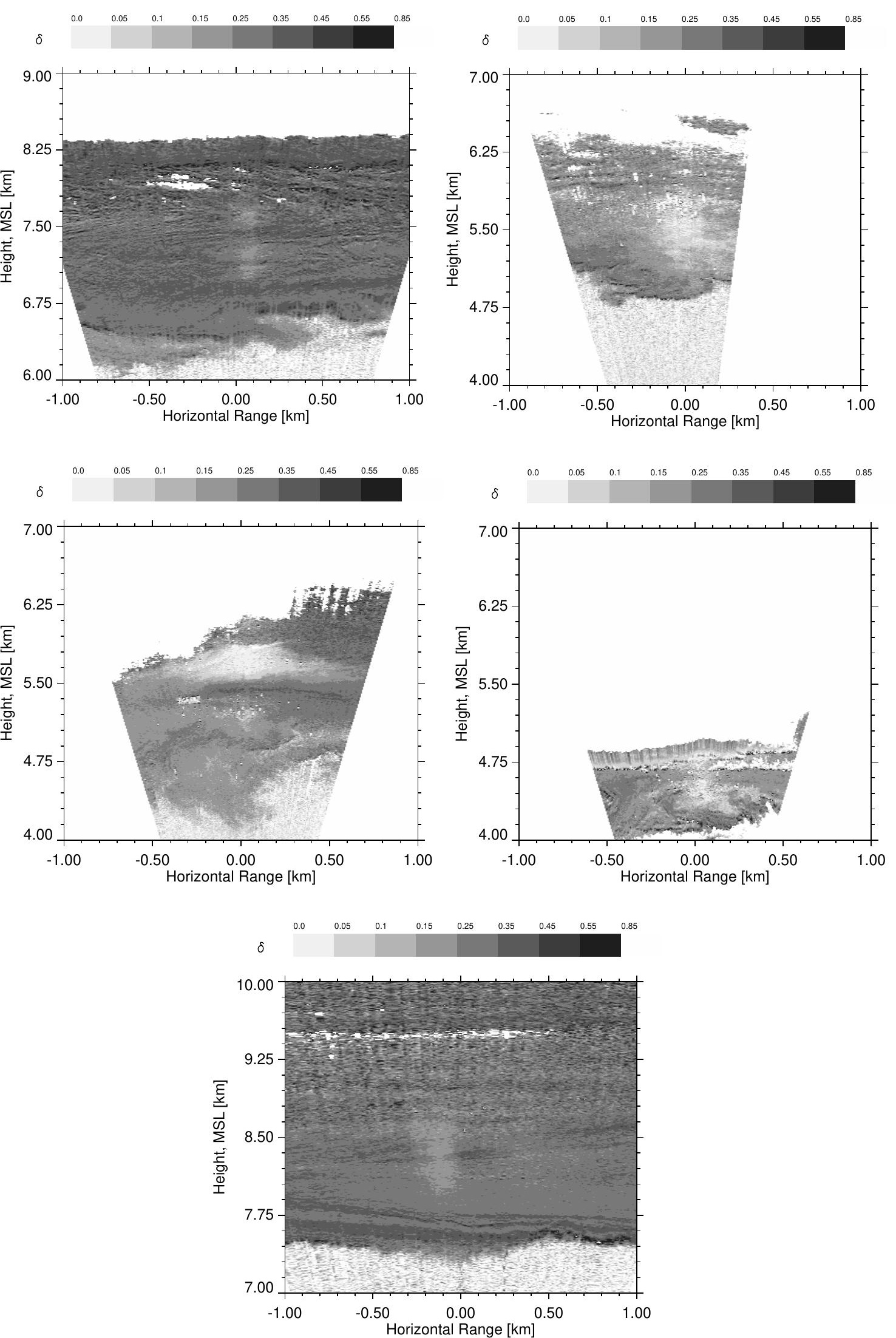


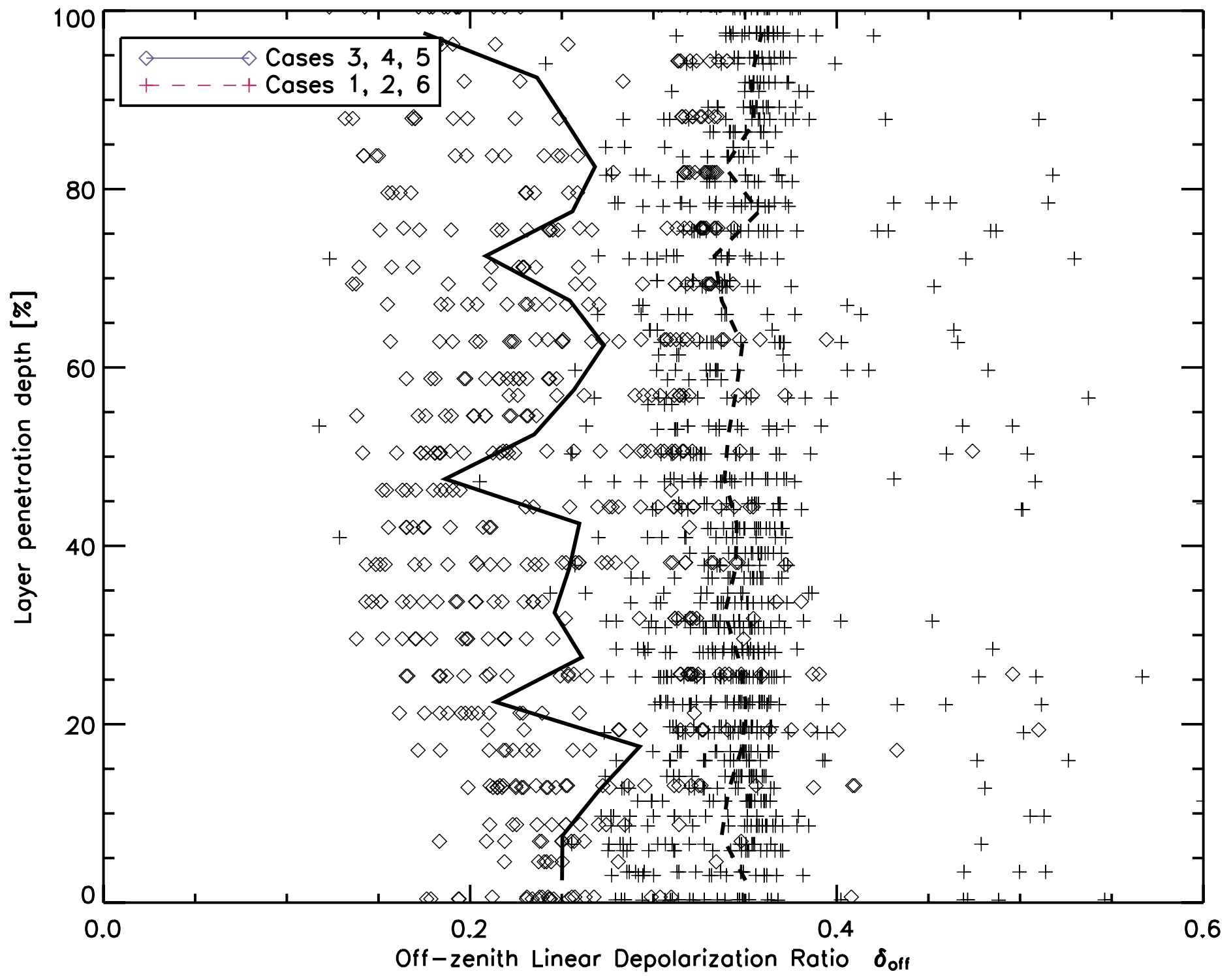



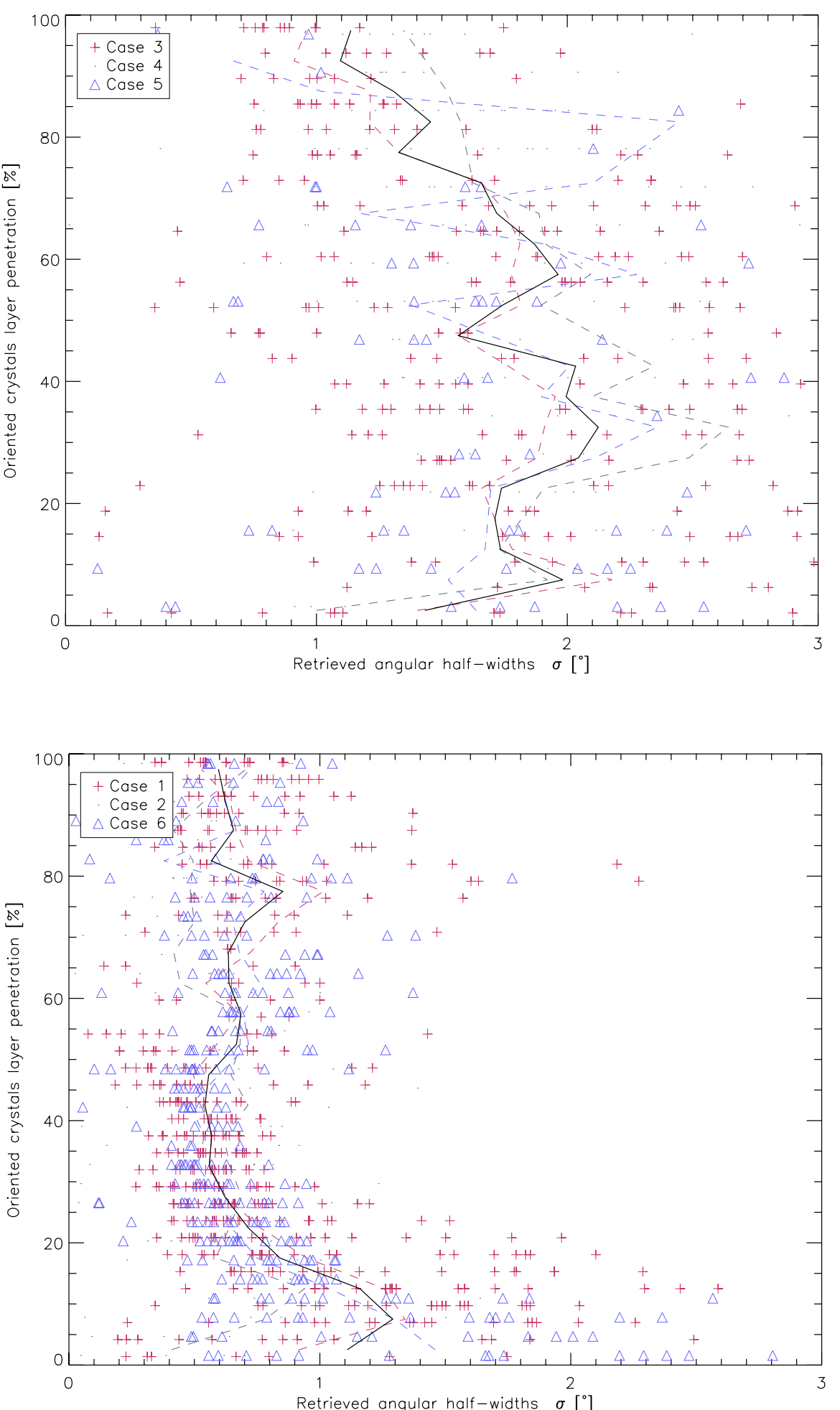
Warm clouds $\left(\mathrm{T}>-20^{\circ} \mathrm{C}\right)$

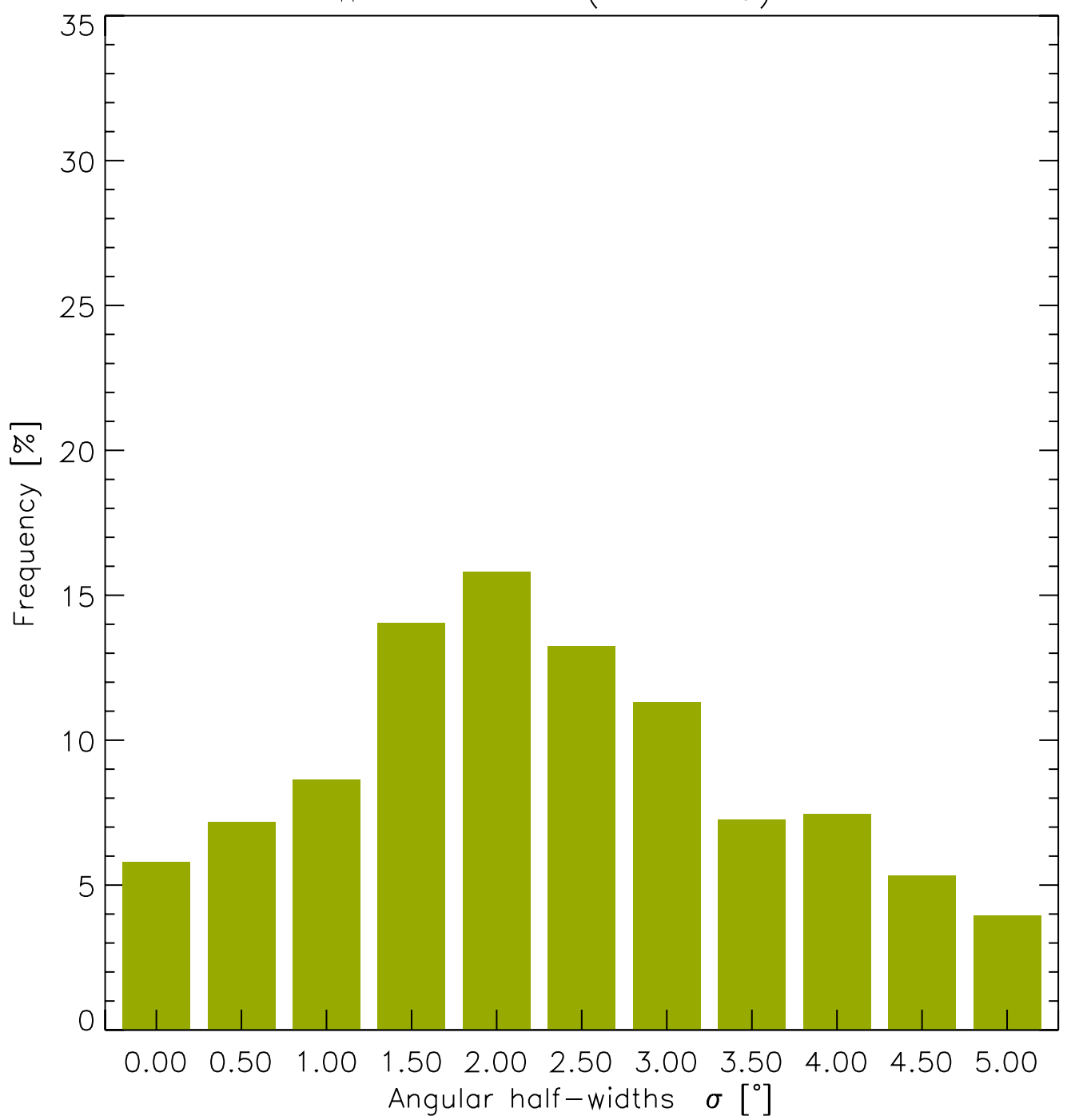

Cold clouds $\left(T<-20^{\circ} \mathrm{C}\right)$

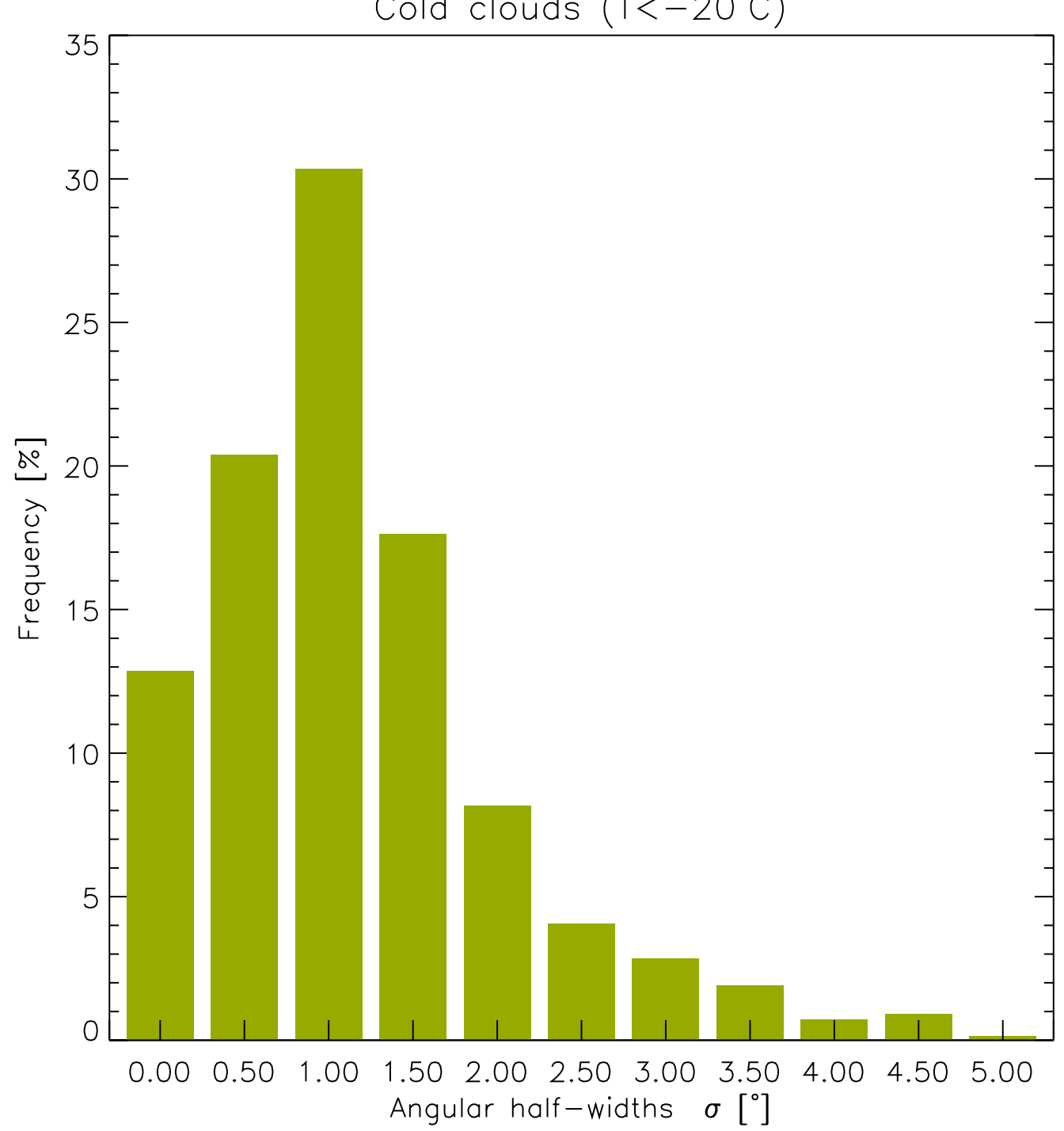




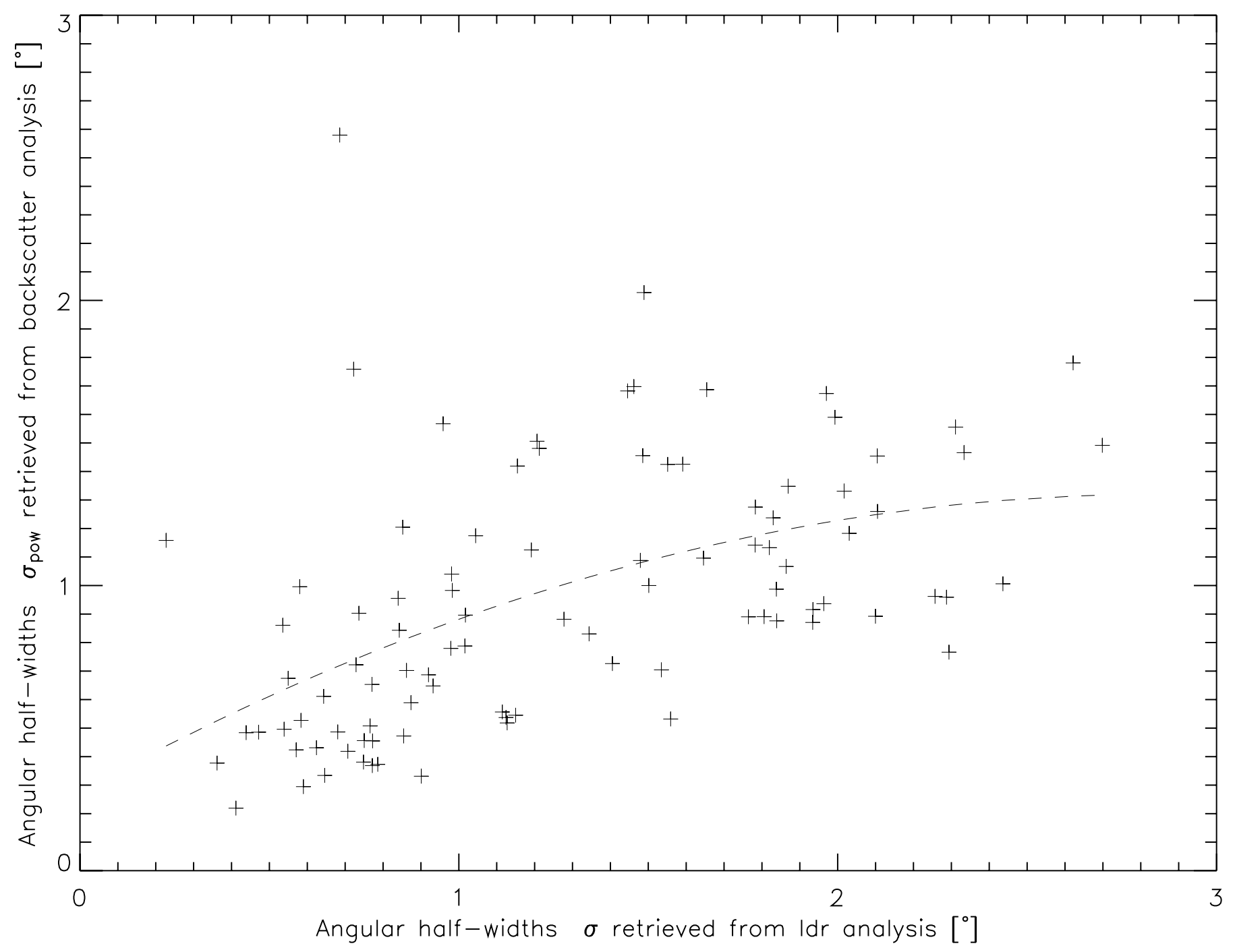

